\title{
Effects of rotation and magnetic fields on the structure and surface abundances of solar-type stars
}

\author{
P. Eggenberger, A. Maeder, and G. Meynet \\ Observatoire de Genève, Université de Genève, \\ 51 Ch. des Maillettes, CH-1290 Versoix, Suisse \\ email: [patrick.eggenberger; andre.maeder; georges .meynet] @unige.ch
}

\begin{abstract}
The effects of shellular rotation on the modelling of solar-type stars (in particular internal structure, evolutionary tracks in the HR diagram, lifetimes and surface abundances) are first examined. Then the effects of a dynamo possibly occuring in the internal stellar radiative zone by imposing nearly solid body rotation are studied. These results are finally discussed in the context of the rotational history of exoplanet host stars and the link between lithium depletion and the presence of exoplanets.
\end{abstract}

Keywords. Stars: rotation, magnetic fields, interiors, abundances

\section{Introduction}

Rotation is one of the key processes that changes the internal structure and global properties of stellar models with a peculiarly strong impact on the physics and evolution of massive stars (see e.g. Maeder 2009). In this work, we first focus on the effects of rotational mixing on the global properties and surface abundances of solar-type stars by comparing stellar models including shellular rotation to non-rotating models. We then investigate the effects of a dynamo in the radiative zone of a solar-type star on the efficiency of rotational mixing by computing models with shellular rotation and the Tayler-Spruit dynamo (Spruit 2002). A comparison between models of solar-type stars including rotation only and both rotation and magnetic fields is made in the framework of a scenario proposed by Bouvier (2008) that relates the enhanced lithium depletion in exoplanet host stars to their rotational history.

\section{Effects of rotation}

To investigate the effects of rotational mixing on the properties of solar-type stars, $1 \mathrm{M}_{\odot}$ models are computed with the Geneva stellar evolution code (Eggenberger et al. 2008). These models are computed with a solar chemical composition as given by Grevesse \& Noels (1993) and a solar calibrated value for the mixing-length parameter. The mainsequence evolution of non-rotating models with and without atomic diffusion of helium and heavy elements is compared to the main-sequence evolution of a rotating model with an initial velocity of $50 \mathrm{~km} \mathrm{~s}^{-1}$ on the zero age main sequence (ZAMS). We adopt the braking law of Kawaler (1988) to account for the magnetic braking undergone by solartype stars when arriving on the main sequence. Two parameters enter this braking law: the saturation velocity $\Omega_{\text {sat }}$ and the braking constant $K$. Following Bouvier et al. (1997), $\Omega_{\mathrm{sat}}$ is fixed to $14 \Omega_{\odot}$ and the braking constant $K$ is calibrated so that a $1 \mathrm{M}_{\odot}$ star with an initial velocity of $50 \mathrm{~km} \mathrm{~s}^{-1}$ on the ZAMS reproduces the solar surface rotational velocity after $4.57 \mathrm{Gyr}$. The rotating model also includes the effects of atomic diffusion 


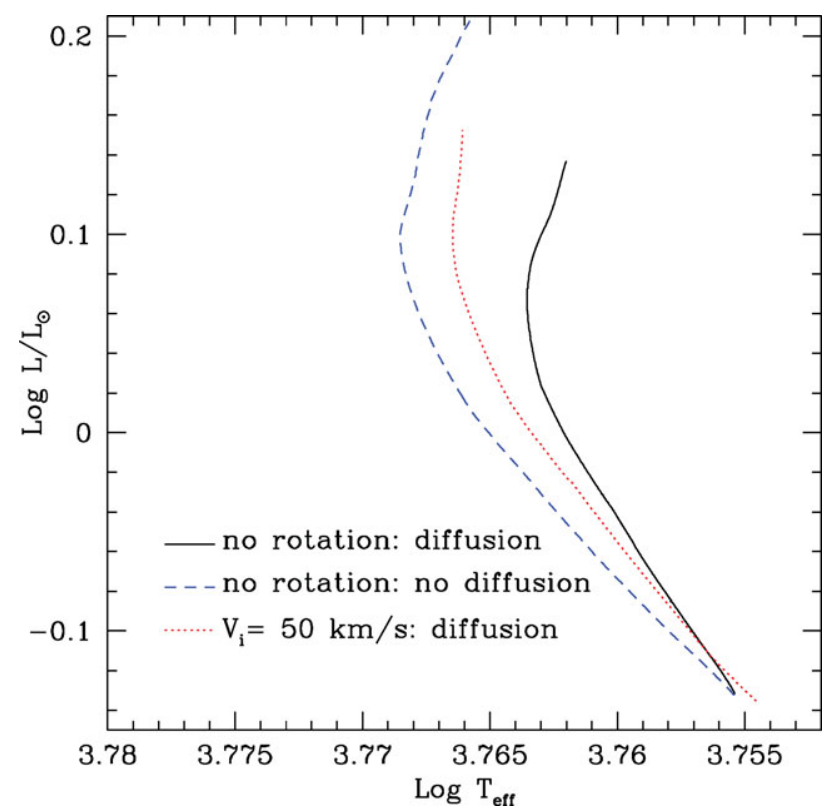

Figure 1. Main-sequence evolution of $1 \mathrm{M}_{\odot}$ models with and without rotation. The continuous and dashed lines correspond to non-rotating models computed with and without atomic diffusion, respectively. The dotted line indicates a rotating model computed with an initial velocity of $50 \mathrm{~km} \mathrm{~s}^{-1}$ on the ZAMS and with atomic diffusion.

of helium and heavy elements. All three $1 \mathrm{M}_{\odot}$ models share therefore exactly the same initial parameters except for the inclusion of shellular rotation and atomic diffusion.

The evolutionary tracks in the HR diagram corresponding to the main-sequence evolution of these models are shown in Fig. 1. By comparing the rotating model (dotted line) to the non-rotating model computed with the same initial parameters except for the inclusion of shellular rotation (continuous line), we see that the rotating model exhibits larger effective temperatures and slightly larger luminosities than the non-rotating one. This results in a shift of the evolutionary track to the blue part of the HR diagram when rotation is included in the computation. Concerning atomic diffusion, Fig. 1 shows that the non-rotating model including atomic diffusion (continuous line) is characterised by lower effective temperatures and luminosities compared to the non-rotating model without atomic diffusion (dashed line). The inclusion of atomic diffusion is thus found to shift the evolutionary track towards the red part of the HR diagram.

These changes observed in the HR diagram can be related to differences in the surface chemical composition of the models. Fig. 2 shows the helium surface mass fraction $Y_{\mathrm{s}}$ as a function of time during the main-sequence evolution for the three models shown in Fig. 1. The model computed without atomic diffusion and rotation (dashed line) exhibits a constant value of the helium surface abundance since no mixing mechanisms are at work in the radiative zone. The inclusion of atomic diffusion leads to a decrease of the helium mass fraction at the stellar surface. By comparing the models including atomic diffusion computed with and without rotation (dotted and continuous lines), we note a lower decrease of the helium surface abundance for the rotating model than for the non-rotating one. Rotational mixing is thus found to counteract the effects of atomic diffusion in the external layers of the star. As a result, rotating models exhibit larger values of helium abundances at the surface. This leads to a decrease of the opacity in 


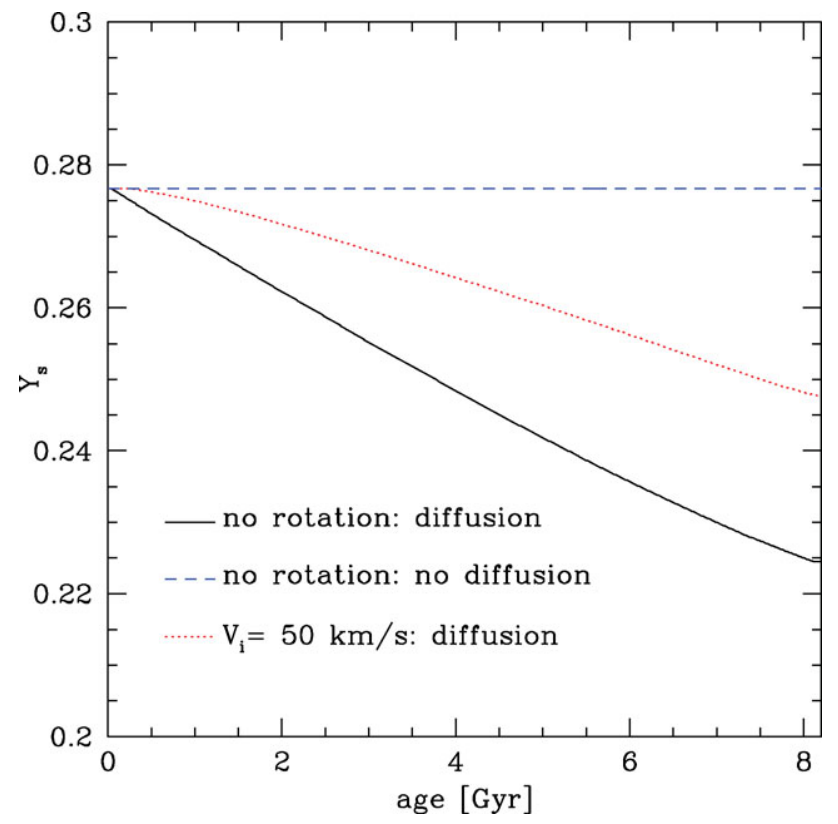

Figure 2. Surface helium mass fraction during the evolution on the main sequence for the $1 \mathrm{M}_{\odot}$ models shown in Fig. 1.

the external layers of the star and explains the shift towards the blue part of the HR diagram observed in Fig. 1.

The effects of rotation are of course not restricted to the external layers of the star. Rotational mixing has indeed a large impact on the properties of the central layers by bringing fresh hydrogen fuel to the central stellar core. As a result, the central hydrogen mass fraction at a given age is found to be larger for rotating models than for models without rotation. The inclusion of atomic diffusion leads to the opposite effect, since a non-rotating model computed with atomic diffusion exhibits a more rapid decrease of the central hydrogen abundance than a model without atomic diffusion. It is interesting to note that the efficiency of rotational mixing relative to atomic diffusion is found to be larger in the central layers of a solar-type star than in its external layers. In the external layers, rotation only reduces the efficiency of atomic diffusion but does not completely counteract these effects (see Fig. 2), while in the central layers the increase of the hydrogen abundance due to rotation is larger than the decrease related to atomic diffusion. Due to rotational mixing, the main-sequence lifetime is then larger for stellar models including rotation.

Rotational effects change the structure of a solar-type star and hence its asteroseismic properties. In particular, the change of the central chemical gradients and the increase of the central hydrogen abundance induced by rotational mixing lead to an increase of the values of the asteroseismic small separation for rotating models compared to non-rotating models (Eggenberger et al. 2006; Eggenberger \& Carrier 2006). The effects of rotation on the external layers are of course reflected in the change of the surface abundances but can also be revealed by asteroseismic measurements. As discussed above, the inclusion of rotation results in a significant increase of the effective temperature. At a given luminosity, this leads to smaller radii for rotating models than for non-rotating models. Consequently, a rotating model will be characterised by a larger mean density and hence a larger value of the asteroseismic mean large separation than a non-rotating model. It is 


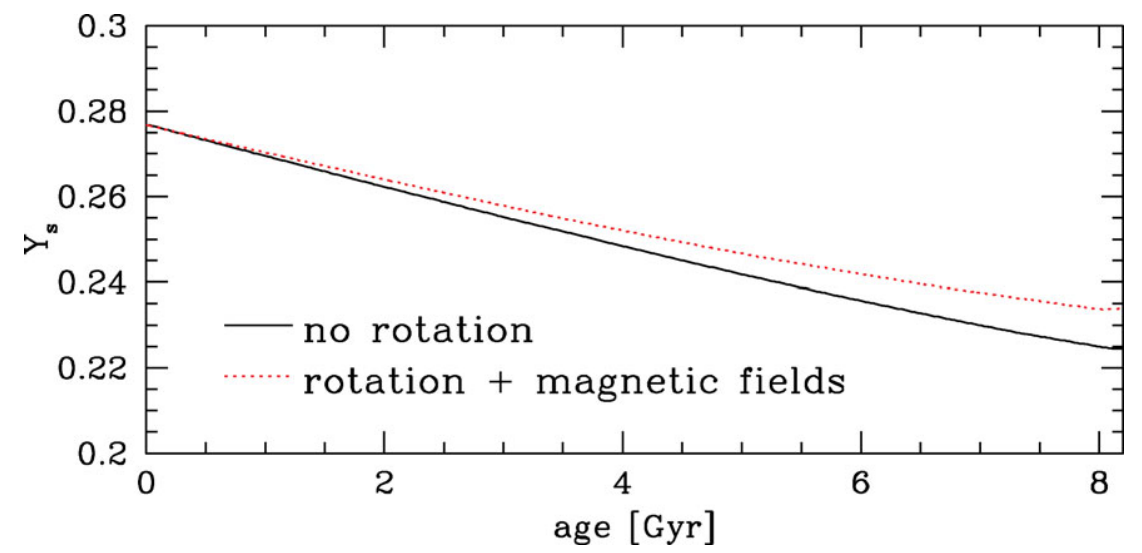

Figure 3. Surface helium mass fraction during the main-sequence evolution of a non-rotating model (continuous line) and a model including rotation and magnetic fields (dotted line). Both models are computed with atomic diffusion of helium and heavy elements.

thus interesting to note that spectroscopic measurements of the surface abundances and asteroseismic measurements can give us valuable insights about the effects of rotational mixing on the properties of solar-type stars.

\section{Effects of magnetic fields}

After the effects of rotation, the effects of magnetic fields and in particular of a dynamo possibly occuring in the radiative zone of the star are studied. These effects are particularly interesting to consider in the context of the internal rotation of the Sun, since models including only shellular rotation predict a rapidly rotating core in disagreement with helioseismic measurements. Models of solar-type stars including both shellular rotation and the Tayler-Spruit dynamo (Spruit 2002) are then computed. We recall here that the theoretical formulation of this dynamo is still a matter of debate (see e.g. Denissenkov \& Pinsonneault 2007; Zahn et al. 2007; Rüdiger et al. 2009). It is however worth investigating the effects of such an efficient process for the transport of angular momentum on the properties of solar-type stars, since models including both the effects of shellular rotation and magnetic fields as prescribed by the Tayler-Spruit dynamo are found to correctly reproduce the helioseismic measurements of the solar rotation profile (Eggenberger et al. 2005).

Figure 3 shows the comparison between the helium surface abundance during the main sequence for a $1 \mathrm{M}_{\odot}$ model including both shellular rotation and the Tayler-Spruit dynamo computed with an initial velocity on the ZAMS of $50 \mathrm{~km} \mathrm{~s}^{-1}$ and the corresponding non-rotating model. Both models include atomic diffusion of helium and heavy elements. The inclusion of both rotation and magnetic fields results in a slight increase of the helium mass fraction at the stellar surface compared to a non-rotating model including only atomic diffusion. This increase is however much lower than for a model computed with rotation only (compare Fig. 2 and Fig. 3). The efficiency of rotational mixing is thus found to be strongly reduced when the effects of the Tayler-Spruit dynamo are taken into account. The near solid body rotation of models including this dynamo leads indeed to a low value of the diffusion coefficient associated to the shear turbulent mixing. The transport of chemicals by shear mixing is thus strongly reduced when the effects of the Tayler-Spruit are included in the computation. Compared to the case with rotation only, we also note a slight increase of the transport of chemicals by the meridional 
circulation for models with magnetic fields. This increase is however much smaller than the strong decrease of the shear turbulent mixing leading to a net decrease of the global efficiency of rotational mixing for a rotating model of a solar-type star computed with the Tayler-Spruit dynamo.

\section{Rotational history of exoplanet host stars}

Finally, the effects of rotation and magnetic fields are briefly discussed in the context of the rotational history of exoplanet host stars and in particular in the framework of a scenario suggested by Bouvier (2008). This scenario is first based on the fact that observations of rotational periodes of young solar-type stars suggest that slow rotators develop a high degree of differential rotation between the radiative core and the convective envelope, while solid-body rotation is favoured for fast rotators (see e.g. Irwin et al. 2007; Bouvier 2008). This result implies that slow rotators can be modelled with shellular rotation only, while fast rotators are modelled with both rotation and the Tayler-Spruit dynamo in order to produce a sufficient internal coupling to ensure solid body rotation. As discussed in the preceding section, this leads to different surface chemical compositions for slow and fast rotators, since the efficiency of rotational mixing is strongly reduced when the effects of magnetic fields are taken into account. Slow rotators will then exhibit lower values of surface lithium abundance than fast rotators.

As discussed by Bouvier (2008), the rotation of the star on the ZAMS depends on the initial velocity of the star and on the disk lifetime during the pre-main sequence. A longer disk lifetime enables the star to lose a larger amount of angular momentum during the pre-main sequence leading to a lower rotation rate on the ZAMS. As mentioned above, slow rotators are characterised by lower surface abundances of lithium than fast rotators. We thus obtain a relationship between the surface lithium abundance and the rotation rate on the ZAMS which seems to be in good agreement with observations in the Pleiades (Soderblom et al. 1993). Moreover, longer disk lifetimes may favor the formation and migration of giant exoplanets. This leads to an interesting relationship between the abundance of lithium and the presence of giant exoplanets. In this scenario, a longer disk lifetime leads indeed simultaneously to a lower lithium abundance (due to the smaller rotation rate on the ZAMS) and a higher probability to detect giant exoplanets. This seems to be in good agreeement with observations of lithium depletion in exoplanet host stars as reported by Israelian et al. (2009).

One may wonder whether other observational trends are predicted in the framework of this scenario. As mentioned in Sect. 2, the effects of rotation on the properties of the central layers of a solar-type star can be revealed by asteroseismic observations and in particular by changes of the small separation. In the scenario outlined here, the efficiency of rotational mixing is larger for slow rotators on the ZAMS than for fast rotators. Since the presence of giant exoplanets is favored for stars with slow rotation rates on the ZAMS, the efficiency of rotational mixing is then predicted to be larger in exoplanet host stars than in stars without planets. This explains the different lithium abundances of stars with and without planets but this also leads to changes in the structure and chemical composition of the central layers and hence to different values of the small separation. In the scenario proposed by Bouvier (2008) we thus expect larger values of the asteroseismic small separation for exoplanet host stars than for stars without planets. It will be particularly interesting to investigate this point in the light of new asteroseismic observations coming from space missions dedicated simultaneously to the search of exoplanets and asteroseismology like CoRoT and Kepler. 


\section{References}

Bouvier, J. 2008, A\&A, 489, L53

Bouvier, J., Forestini, M., \& Allain, S. 1997, A\&A, 326, 1023

Denissenkov, P. A. \& Pinsonneault, M. 2007, ApJ, 655, 1157

Eggenberger, P. \& Carrier, F. 2006, A\&A, 449, 293

Eggenberger, P., Carrier, F., Maeder, A., \& Meynet, G. 2006, Memorie della Societa Astronomica Italiana, 77, 309

Eggenberger, P., Maeder, A., \& Meynet, G. 2005, A\&A, 440, L9

Eggenberger, P., Meynet, G., Maeder, A., et al. 2008, Ap\&SS, 316, 43

Grevesse, N. \& Noels, A. 1993, in Origin and evolution of the elements: proceedings of a symposium in honour of H. Reeves, held in Paris, June 22-25, 1992. Edited by N. Prantzos, E. Vangioni-Flam and M. Casse. Published by Cambridge University Press, Cambridge, England, 1993, p.14, ed. N. Prantzos, E. Vangioni-Flam, \& M. Casse, 14

Irwin, J., Hodgkin, S., Aigrain, S., et al. 2007, MNRAS, 377, 741

Israelian, G., Delgado Mena, E., Santos, N. C., et al. 2009, Nature, 462, 189

Kawaler, S. D. 1988, ApJ, 333, 236

Maeder, A. 2009, Physics, Formation and Evolution of Rotating Stars, ed. A. Maeder

Rüdiger, G., Gellert, M., \& Schultz, M. 2009, MNRAS, 399, 996

Soderblom, D. R., Jones, B. F., Balachandran, S., et al. 1993, AJ, 106, 1059

Spruit, H. C. 2002, A\&A, 381, 923

Zahn, J., Brun, A. S., \& Mathis, S. 2007, A\&A, 474, 145 